\section{EXCEPTIONAL QUALITY, OUTSTANDING DESIGN}

Evident supplies high quality custommade ExamVision loupes supported by their special dental knowledge and optical experience.

Available in two styles, three frame colours, three frame sizes and four

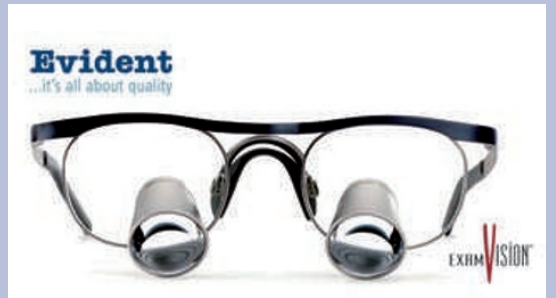

magnifications, each ExamVision loupe is individually crafted to your personal measurements and requirements. Whether you normally wear glasses or not, ExamVision's lightweight, highdefinition loupes will vastly improve your detailed vision of the whole treatment area, whilst also improving your comfort, making it easier to work and reducing stress.

Evident supply the very best precision products, combining exceptional quality and service with outstanding design.

Reader response number 62

\section{MAINTAIN CRESTAL BONE}

The Laser-Lok 3.0 from BioHorizons is the first $3 \mathrm{~mm}$ implant that incorporates Laser-Lok technology to create a biologic seal and maintain crestal bone on the implant collar. Designed specifically for limited spaces in the aesthetic zone, the Laser-Lok 3.0 dental implant comes with a broad array of prosthetic options making it the perfect choice for high profile cases.

Laser-Lok 3.0 dental implants take advantage of the latest engineering techniques to provide a strong, highly aesthetic design for extremely small spaces. Each implant comes with a $1 \mathrm{~mm}$ cover cap/healing abutment. There is also a choice of final abutments available to the clinician to suit relevant clinical conditions and optimise aesthetics. Delivery to the mouth can be achieved by inserting a 0.050 " hex driver into the abutment screw that is pre-assembled into the abutment, saving time and additional assembly.

Laser-Lok is a patented lasermachined surface treatment with over 15 years of in vitro, animal and human studies. Laser-Lok microchannels are a series of precision-engineered 8 and 12 micron grooves on the collar of dental implants. This patented laser surface is unique within the industry as the only surface treatment shown to attach and retain both hard and soft tissue.

Reader response number 64

\section{GDP IMPLANT REVOLUTION}

General dental practitioners from all over the UK are starting to restore dental implants for their own patients through a nationwide campaign of local events backed by DENTSPLY Friadent and available at www.dentists4implants.com.

The GDP implant restoration training programme began last year, and has accelerated during 2010 to include more than 50 local 'R£LAX' events from Scotland to Cornwall. The complete package is provided free, including three hours CPD, a mini prosthetics kit for restoring the first case, a CD on

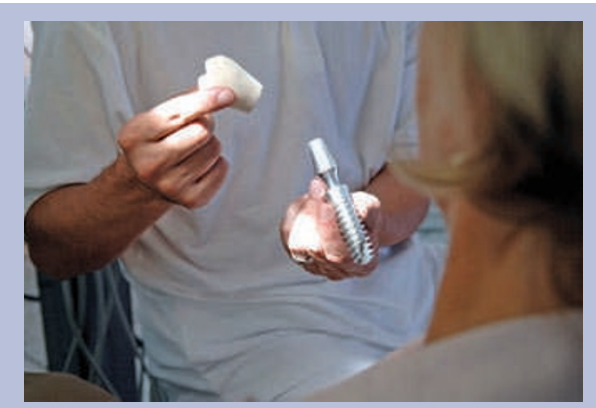

impressions and placement, Practice Builder patient information, in-surgery support for first case, local laboratory support and a $£ 100$ education voucher.

Implant treatment can improve clinical outcomes for patients and expand income options for their dentist. Getting involved with restoration of straightforward cases can help strengthen patient relationships and enhance practice reputation, and restoring simple dental implant cases is quick and profitable. Minimal additional training and equipment are needed by most general dental practitioners.

If the list of forthcoming 'R£LAX' events does not include a location/ date which is convenient, DENTSPLY Friadent can still help. The company can also arrange individual implant restoration training sessions or set up a personal 'R£LAX' chairside tutorial with a local implant dentist.

Reader response number 65 